\title{
Avaliação morfométrica da região apical após a utilização de sistemas oscilatório e rotatório
}

\section{Morphometric evaluation of the apical region after the use of oscillatory and rotary syeterns}

lanara Janayna Leal Ferraz*

Naiana Costa de Brito*

Larissa Cordeiro Cavalcante ${ }^{* *}$

Isadora Mello Vilarinho Soares ${ }^{* * *}$

Carmen Milena Rodrigues Siqueira Carvalho ${ }^{* * *}$

\section{Resumo}

Objetivo: este trabalho avalia comparativamente os sistemas oscilatório e contínuo de preparo de canais, a partir da análise morfométrica, da região apical de molares preparadas com limas manuais flexofile acionadas a motores pneumático (oscilatório) e elétrico (rotatório), acopladas ao sistema ProTaper Universal. Materiais e métodos: vinte raízes de molares inferiores humanos extraídos com curvaturas aproximadas de 30 graus foram selecionadas e inicialmente submetidas a radiografias. As imagens radiográficas foram digitalizadas e por meio do Software Image tool determinados a área e o perímetro da região apical. Em seguida, as raízes foram divididas aleatoriamente em dois grupos: no grupo $I$, as raízes foram instrumentadas pelo sistema oscilatório e, no grupo II, pelo sistema rotatório. Os terços cervical e médio foram preparados com Gates Glidden 1 e 2. Após o preparo químico-mecânico, novas radiografias foram realizadas para a determinação da nova área e do novo perímetro. Os resultados foram analisados estatisticamente no programa Statistical Package for the Social Sciences (SPSS) da versão 20.0, com nível de significância de 5\% $(p<0,05)$. Resultados: o sistema rotatório apresentou diferença estatisticamente significativa quanto à área antes e após o preparo químico mecânico $(p<0,05)$ e não ocorreu diferença quanto ao perí- metro. Não foram observados desvios apicais atribuídos à maior proporcionalidade entre o preparo apical e a dilatação dos terços médio e cervical realizado previamente. Conclusão: ambos os sistemas apresentaram um preparo apical aceitável, porém o sistema oscilatório, empregando limas flexofile desgastou menor espessura dentinária quando comparadas ao sistema ProTaper Universal.

Palavras-chave: Tratamento do canal radicular. Ápice dentário. Instrumentos odontológicos. Endodontia.

\section{Introdução}

O preparo do canal radicular é um dos momentos mais críticos da terapia endodôntica e está intimamente relacionado com o processo de desinfecção e das etapas subsequentes. Seu objetivo é eliminar tecido pulpar, bactérias e outros produtos derivados; além de proporcionar uma forma adequada e permitir uma posterior obturação hermética dos canais radiculares ${ }^{1}$.

Graduada em Odontologia pela Universidade Federal do Piauí (UFPI), Teresina, Piauí, PI, Brasil.

Graduanda em Odontologia pela Universidade Federal do Piauí (UFPI), Teresina, Piauí, PI, Brasil.

Acadêmica do Programa de Pós-Graduação em Odontologia da Universidade Federal do Piaú́ (UFPI), Teresina, Piauí, PI, Brasil.

**** Professora Doutora do Departamento de Patologia e Clínica Odontológica do Centro de Ciências da Saúde (CSS) da Universidade Federal do Piauí (UFPI), Teresina, Piauí, PI, Brasil. 
As técnicas manuais foram as primeiras a serem utilizadas nesse fim, e, até hoje, são extremamente difundidas. Porém, essa instrumentação apresenta limitações no que se refere à limpeza dos canais radiculares, bem como à possibilidade da ocorrência de iatrogenias durante o preparo (transporte do canal, formação de degraus, perfurações) ${ }^{2}$. Devido a isso, muitas mudanças ocorreram no intuito de permitir o desenvolvimento de novas técnicas que possibilitassem maior precisão e menor tempo clínico. Sistemas automatizados foram desenvolvidos e esses se dividem em três tipos: sônicos, ultrassônicos e mecânicos, cujo propósito é cumprir, na íntegra, os objetivos do tratamento endodôntico, minimizando os riscos de acidentes e diminuindo o tempo gasto nos preparos ${ }^{3}$.

Os instrumentos de níquel-titânio, desenvolvidos em 1988, possibilitaram a introdução de técnicas rotatórias que foram melhoradas significativamente nos últimos anos, permitindo um preparo mais racional dos canais radiculares e propiciando maiores índices de sucesso ${ }^{4-6}$. Junto com a melhoria da instrumentação rotatória, o sistema oscilatório foi reintroduzido à prática odontológica, e pode ser acionado por motores elétricos ou pneumáticos, com a vantagem de poder ser utilizado tanto com limas de aço inoxidável, como de $\mathrm{NiTi}^{7}$.

Diante da complexidade do tratamento endodôntico principalmente na frágil região apical, faz-se evidente a necessidade de avaliar ensaios e recursos. Assim, a pesquisa realizada tem por objetivo avaliar comparativamente os sistemas oscilatório e rotatório de preparo de canais a partir da análise morfométrica da região apical de molares preparadas com limas manuais Flexofile ${ }^{\circledR}$ (Denteply Maillefer,Ballaigus,Suiça) acionadas a motores pneumático (oscilatório), e elétrico (rotatório contínuo) acopladas ao sistema Protaper Universal ${ }^{\circledR}$ (Dentsply Maillefer, Ballaiguies, Suíça).

\section{Materiais e métodos}

Este trabalho de pesquisa teve seu projeto aprovado pelo Comitê de Ética em Pesquisa CEP/ UFPI (0187.0.045.000-09).

Foram obtidas vinte raízes de molares inferiores humanos extraídos, que apresentavam como características anatômicas curvaturas aproximadas de 30 graus, e confirmadas por tomadas radiográficas no sentido vestíbulo-lingual ${ }^{8}$. Realizou-se o acesso às câmaras coronárias por intermédio de brocas específicas para esse fim e, em seguida, os dentes foram imersos em solução de hipoclorito de sódio a 2,5\% (Fórmula \& Ação, São Paulo, Brasil), em que permaneceram por 72 horas sendo posteriormente autoclavados. Os dentes foram, então, lavados em água corrente e armazenados em solução de soro fisiológico $0,9>\mathrm{ADV}$ Farma $^{\circledR}$, (Nova Odessa-SP, Brasil) para hidratação, por um período de sete dias.
Decorrido esse tempo, os espécimes foram incluídos em resina acrílica transparente, utilizando-se uma base de plástico adaptada de forma de gelo, permanecendo exposta a face oclusal. Foi então realizada a odontometria dos canais com a lima K-file 10 (Dentsply Maillefer, Ballaigues, Suiça) para obtenção do comprimento real de trabalho, padronizando $1 \mathrm{~mm}$ aquém do ápice.

A seguir, aplicou-se uma substância contraste na proporção de três partes de iodofórmio para uma de propilenoglicol. A injeção foi realizada com o auxílio de seringa descartável, posicionando a agulha no interior dos canais radiculares. Para um melhor preenchimento de todo o canal, utilizou-se limas 10 k-file, manualmente, ao longo do comprimento de trabalho.

A padronização das tomadas radiográficas foi obtida com o auxílio de um mecanismo centralizador que permitiu o posicionamento do cone do aparelho de raios $\mathrm{X}$ em exata perpendicularidade ao plano e, consequentemente, ao bloco de resina com os dentes, minimizando o risco de distorções das imagens. O tempo de exposição foi de 0.5 segundos. Dessa forma, as amostras foram posicionadas e radiografadas no sentido vestíbulo-lingual. Posteriormente à captação das imagens radiográficas, foi realizada a digitalização dessas, mas com o auxílio de uma máquina fotográfica Sony H11 a uma distância focal padronizada de $20 \mathrm{~cm}$. Essas foram transferidas para o Software Image tool (Health Science Center, Universidade do Texas, Texas, Estados Unidos), cujo processamento de imagens possibilitou determinar por meio do sistema a região apical antes e após o preparo. E, com o sistema de réguas, foi possível mensurar exatamente a extensão dos terços apicais selecionados, obtendo-se, desse modo, medidas em milímetros, visto ser uma imagem virtual. Em seguida, foram processados os cálculos referentes à área (pixels) e ao perímetro $(\mathrm{mm})$. A leitura foi realizada por um único observador cego.

Após as mensurações realizadas anteriormente, os espécimes foram divididos aleatoriamente em dois grupos, com dez amostras em cada. No grupo I, os canais foram preparados de acordo com a técnica do preparo cervical com o auxílio de brocas Gates-Glidden (Dentsply Maillefer, Ballaigues, Suiça), e ampliação do batente apical correspondente a lima K-file 30 (Dentsply Maillefer, Ballaigues, Suiça) em um sistema oscilatório NSK (NSK, Tóquio, Japão) acoplado às limas flexofile (Dentsply Maillefer, Ballaigues, Suiça), de acordo com o comprimento de trabalho já determinado. Inicialmente, foi realizado o preparo dos terços cervical e médio com broca Gates-Glidden número 1 montada em um contra-ângulo acoplado a um micromotor (Kavo, São Paulo, Brasil). Quando a broca apresentou-se livre no interior dos canais, foi utilizada a Gates-Glidden número 2. Concluído o preparo dos terços cervical e médio, foi realizada a instrumentação oscilatória 
na região apical com limas Flexofile de números 15 , 20, 25, 30 no comprimento de trabalho, todas de primeiro uso, pré-curvadas. Logo após, realizou-se o escalonamento progressivo anatômico. A cinemática aplicada constituiu de leve pressão apical, movimento de vai e vem e apoio em direção à parede externa da curvatura, denominada também de zona de resistência.

No grupo II, empregou-se o Sistema ProTaper Universal $^{\circledR}$, (Dentsply Maillefer, Ballaigues, Suiça) acoplado a motor X-Smart (Dentsply Maillefer, Ballaigues, Suiça), com velocidade $250 \mathrm{rpm}$ e torque $3 \mathrm{~N}$ para realização da técnica cérvico-apical. Após o preparo dos terços cervical e médio com as brocas Gates-Glidden número 1 e 2, a porção apical foi preparada com os instrumentos F1, F2 e F3 e, assim, pode-se padronizar em 0,03 centésimos de mm o batente apical.

O preparo dos canais foi realizado em presença de solução de hipoclorito de sódio a $0,5 \%$ (Fórmula \& Ação, São Paulo, Brasil), com irrigação de $5 \mathrm{~mL}$ a cada troca de instrumento. Ao término dos preparos, os canais foram novamente preenchidos com a pasta de iodofórmio e propilenoglicol para a obtenção de novas radiografias e foi obtido novamente a área (pixels) e o perímetro ( $\mathrm{mm})$. Os dados obtidos antes e após o preparo químico mecânico foram então tabulados e submetidos à análise estatística pelo programa Statistical Package for the Social Sciences (SPSS) versão 20.0 para Windows (SPSS Inc., Illinois, EUA), adotando nível de significância de $5 \%(\mathrm{p}<0,05)$.

\section{Resultados}

Após a tabulação dos dados (Tabela 1), foram obtidas as médias das diferenças de área e perímetro antes e após o preparo químico mecânico com os sistemas oscilatório e rotatório, e essas foram comparadas pelo teste $t$-student (Tabela 2 ).

Tabela 1- Média e desvio padrão da área (pixels) e do perímetro ( $\mathrm{mm}$ ) dos canais antes e após o preparo químico-mecânico de acordo com os sistemas utilizados para instrumentação

\begin{tabular}{l|r|r}
\cline { 2 - 3 } & Oscilatório média (DP) & Rotatório média (DP) \\
\hline Área & & \\
Antes & $2.294,2(287,2)$ & $3.010,0(631,1)$ \\
Após & $8.894,2(1442,8)$ & $11.446,3(2016,4)$ \\
Perímetro & & \\
Antes & $48,43(6,1)$ & $51,11(0,6)$ \\
Após & $52,77(6,8)$ & $55,12(1,6)$ \\
\hline Fonte: dos autores.
\end{tabular}

Os resultados mostraram que há diferença estatisticamente significativa $(\mathrm{p}<0,05)$ em relação à área antes e após o PQM, utilizando-se o sistema rotatório para a instrumentação, não ocorrendo isso em relação ao perímetro.
Tabela 2 - Comparação das médias das diferenças de área (pixels) e de perímetro $(\mathrm{mm})$ antes e após o preparo químico mecânico de acordo com o tipo de instrumentação empregado. Teresina-PI, 2014

\begin{tabular}{l|r|r|r}
\cline { 2 - 3 } & Oscilatório & Rotatório & \multicolumn{1}{c}{$P^{*}$} \\
\hline $\begin{array}{l}\text { Diferença da área } \\
\text { antes e após o preparo }\end{array}$ & $6.600,0^{\mathrm{a}}$ & $8.436,0^{\mathrm{b}}$ & 0,037 \\
$\begin{array}{l}\text { Diferença do perímetro } \\
\text { antes e após o preparo }\end{array}$ & $4,34^{\mathrm{a}}$ & $4,01^{\mathrm{a}}$ & 0,72 \\
\hline
\end{tabular}

Fonte: dos autores.

* teste $t$-student

Letras diferentes, em uma mesma linha, diferem estatisticamente por meio do teste $t$-student $(p<0,05)$.

\section{Discussão}

O uso de canais simulados em blocos de resina, por um lado, permite uma comparação eficaz direta, porque a forma, o tamanho, o cone, e a curvatura são padronizados. Por outro, a resiliência do material é diferente da dentina, o que poderia ocasionar resultados não confiáveis e, além disso, a maioria dos erros na preparação do canal radicular ocorre em canais curvos, fator esse decisivo na escolha de dentes naturais para o trabalho, pois representa realidade anatômica ${ }^{1,9}$.

A manutenção da forma cônica e uniforme durante a instrumentação, incluindo a completa remoção do conteúdo orgânico do interior do canal radicular, deve obedecer aos princípios básicos de limpeza e de modelagem. Tal fato, não implica em grandes dificuldades quando o preparo refere-se a canais retos. Porém, em canais curvos essa afirmativa pode ser questionada, uma vez que, dentre as dificuldades dessa etapa, há um indiscutível incremento da possibilidade de ocorrência de erros, além da possibilidade de desgaste excessivo das paredes do canal durante o preparo ${ }^{10-13}$.

Neste experimento, optou-se em avaliar técnicas automatizadas em raízes de molares inferiores, uma utilizando limas manuais Flexofile e micromotor pneumático com movimento oscilatório, comparando-os aos instrumentos de NiTi ProTaper ${ }^{\circledR}$ acionadas a motor elétrico, por serem dois grandes avanços no campo da Endodontia que permitem o preparo de um canal radicular mais fácil e rápido, sendo o sucesso do tratamento mais previsível do que com o uso de instrumentos manuais ${ }^{14}$.

Foi eleito o terço apical do canal radicular para a aplicação de análises geométricas, tendo em vista a grande importância dessa região para o tratamento endodôntico, e por ser uma área crítica no que se refere ao preparo ${ }^{15}$. Com relação ao cálculo da área na região apical dos canais radiculares, a média dos valores encontrados revelou um maior desgaste nessa região pelo sistema ProTaper ${ }^{\circledR}$ que pelo sistema oscilatório. A cinemática proporcionada pela preparação oscilatória permite um bom desempenho dos instrumentos nas paredes do canal radicular, resul- 
tando em um adequado preparo do espaço anatômico de forma menos invasiva ${ }^{9}$. Porém, é necessária a instrumentação de toda a área, pois uma pequena área do canal não instrumentada pode abrigar inúmeras toxinas e microrganismos, comprometendo o resultado final do tratamento endodôntico ${ }^{16}$.

No que se refere ao perímetro, esse foi definido como a somatória das alturas e larguras dos pixels localizados na periferia da figura. Essa grandeza pode sugerir dados significantes, como, por exemplo, a aquisição de valores positivos após a ampliação do canal. Porém nesse quesito, não foram observadas diferenças estatisticamente significativas entre as médias dos grupos rotatório e oscilatório.

O desgaste inicial realizado pelas brocas Gates-Glidden, em ambos os grupos, possibilitou a ação dos instrumentos mais livremente no interior do canal, o que levou a não ocorrência de fraturas e desvios apicais durante o preparo. Comparativamente, ao analisarem o Protaper Manual ${ }^{\mathrm{TM}}$ e os Sistemas Rotatórios ProTaper ${ }^{\mathrm{TM}}$ e $\mathrm{RaCe}^{\mathrm{TM}}$, Aguiar e Câmara $^{17}$ (2006) não observaram a ocorrência de desvios no terço apical em molares inferiores humanos por meio da superposição radiográfica.

Ademais, o uso de brocas Gates-Glidden propiciou excelente rendimento do sistema Protaper, suavizando a ação de seus instrumentos, e inferindo-lhes maior liberdade de ação, o que favoreceu melhor e mais rápida atuação destes, após o preparo da entrada dos canais radiculares e a retificação dos terços cervical e médio ${ }^{18}$. Essa observação é extremamente interessante para um sistema que apresenta movimento rotatório contínuo e utiliza instrumentos de níquel-titânio, os quais apresentam risco potencial de fratura ${ }^{5}$.

López et al. ${ }^{19}$ (2008) demonstraram que a instrumentação com rotação contínua com instrumentos \#30, \#35 e \#40 foi eficaz no preparo do canal radicular, em termos de se evitar desvio apical, sendo que o preparo com instrumentos \#35 e \#40 em movimento oscilatório provocaram desvio apical significativo. Tolomelli e Campos $^{20}$ (2005), ao comparar três sistemas motorizados de instrumentação de canais radiculares, encontraram que o sistema ProTaper produziu preparos mais regulares, sem desvios e com conicidade mais progressiva, atribuída à melhor proporcionalidade entre a conicidade do preparo apical e a dilatação dos terços médio e cervical. A análise entre preparos, utilizando limas manuais flexofile e ProTaper automatizado, realizados por Aznar et al. ${ }^{21}$ (2006) indicou não haver diferenças estatisticamente significativas entre os grupos. Porém, ressalva-se que o grupo rotatório apresentou menor média de desvio.

Neste estudo, optou-se por utilizar as limas flexofiles acionadas a motor pneumático por serem limas de fácil acesso e, segundo os achados de Otoboni Filho ${ }^{22}$ (2006), após avaliarem o desgaste produzido nas paredes e o desvio apical em canais curvos simulados em blocos de acrílico, demonstraram que não existem diferenças significativas entre prepa- ros, utilizando sistema de rotação alternada (oscilatório) e diferentes limas de aço inoxidável: Flex-R, Flexofile e Dyna-Flex acionadas pelo Endo-Gripper.

A análise desses sistemas por tomografia computadorizada, um dos métodos recentes de avaliação dessas técnicas, indicou a técnica rotatória como a mais segura, visto que a técnica oscilatória apresentou maior remoção de dentina na região da curvatura ${ }^{7}$. Porém, observou-se que o ProTaper desgastou maior espessura dentinária, verificadas quando se obteve médias estatisticamente significativas em relação à área. Tais divergências podem ser justificadas por fatores externos, visto que, além das técnicas utilizadas, a anatomia do canal radicular, aliada à experiência e à capacidade do operador durante o preparo também podem interferir no resultado e consequente desgaste excessivo de dentina em áreas críticas ${ }^{1}$.

Ressalva-se que, apesar de um maior desgaste dentinário, o sistema ProTaper foi eficaz no preparo apical de canais curvos, e não ocorreram deformidades em relação ao perímetro percorrido por ambos os grupos comparativamente.

\section{Conclusão}

Ambos os sistemas apresentaram um preparo apical aceitável, porém o sistema oscilatório que empregou limas Flexofile, desgastaram menor espessura dentinária quando comparadas ao sistema rotatório.

\section{Abstract}

Objective: to comparatively assess the oscillatory and continuous systems for canal preparation from the morphometric analysis of the apical region of molars prepared with hand Flexofiles driven by pneumatic motors (oscillatory) and electric motors (rotary) coupled to ProTaper Universal system. Materials and Methods: twenty roots of extracted human mandibular molars with approximate curvatures of 30 degrees were selected and initially subjected to radiographs. Radiographs were scanned, and the area and perimeter of the apical region were determined by the Software Image tool. Then the roots were randomly divided into two groups: in group I, the roots were instrumented by the oscillatory system, and in group II, by the rotary system. The cervical and middle thirds were prepared with Gates Glidden 1 and 2. After chemo-mechanical preparation, new radiographs were taken to determine the new area and perimeter. The results were statistically analyzed using the Statistical Package for the Social Sciences (SPSS) version 20.0, with a significance level of 5\% ( $p$ $<0.05)$. Results: the rotary system presented statistically significant difference regarding the area before and after chemical and mechanical preparation $(p<0,05)$, and there was no difference regarding the perimeter. Apical deviation was not observed, which is attributed to the greater proportionality between apical preparation and dilation of middle and cervical thirds, previously 
performed. Conclusion: both systems presented an acceptable apical preparation, however Flexofiles' oscillatory system caused less wear on dentin thickness when compared to the ProTaper Universal system.

Keywords: Root Canal Therapy. Tooth Apex. Dental Instruments. Endodontics.

\section{Referências}

1. Muñoz E, Forner L, Llena C. Influence of operator's experience on root canal shaping ability with a rotary nickeltitanium single-file reciprocating motion system. J Endod 2014; 40(4):547-50.

2. Gonçalves SB, Brosco VH, Bramante CV. Análise comparativa entre instrumentação rotatória, manual e associação de ambas no preparo de canais achatados. J Appl Oral Sci 2001; 11(1): 35-9.

3. Limongi O, Ramos IFA, Troian CH, Vanni JR, Albuquerque DS, Baratto Filho F. Análise in vitro do desvio apical observado no sentido proximal produzido por dois sistemas de rotação alternada: endo-gripper e M4. J Bras Endodontia 2004; 5(16):67-72.

4. Walia H, Brantley WA, Gerstein H. An initial investigation of the bending and torsional properties of nitinol root canal files. J Endod 1988; 14(7):346-51.

5. Zhou HM, Shen Y, Zheng W, Li L, Zheng YF, Haapasalo M. Mechanical properties of controlled memory and superelastic nickel-titanium wires used in the manufacture of rotary endodontic instruments. J Endod 2012; 38(11):1535-40.

6. Lima MCD, Silva PGP, Maia SMAS. Verificação da ocorrência de desvios durante a instrumentação dos canais radiculares: técnica manual $\mathrm{X}$ técnica rotatória: estudo in vitro. Odontol Clín Cient 2002; 1(3):193-6.

7. Hartmann MSM, Fontanella VRC, Vanni JR, Fornari VJ, Barletta FB. CT evaluation of apical canal transportation associated with stainless steel hand files, oscillatory technique and ProTaper rotary system. Braz Dent J 2011; 22(4):288-93.

8. Schneider SWA. A comparison of canal preparations in straight and curved root canals. Oral Surg Oral Med Oral Pathol 1971; 32(2):271-5.

9. Geraldes PF, Aguirre GM, Rivas GJC, Nogueira I, Bonetti-Filho I, García PC et al. Efectividad de las técnicas rotatoria y oscilatoria en la preparación de conductos radiculares de conformación ovalada. Acta odontol venez 2007; 45(4):528-33.

10. Schilder H. Cleaning and shaping root canal. Dent Clin North Am 1974; 18(2):269-96.

11. Abou-Rass M, Frank AL, Glick DH. The anticurvature filling method to prepare the curvature root canal. J Am Dent Assoc 1980; 101(5):792-4.

12. Paiva JG, Antoniazzi JH. Endodontia: bases para a prática clínica. 2. ed. São Paulo: Artes Médicas; 1991.

13. Quackenbush LE. Entation of the curved canal. Dent Update $1987 ; 14(2): 75-9$.

14. Cheung GS, Liu CS. A retrospective study of endodontic treatment outcome between nickel-titanium rotary and stainless steel hand filing techniques. J Endod 2009; 35(7):938-43.

15. Mickel AK, Chogle S, Liddle J, Huffaker K, Jones JJ. The role of apical size determiniation and enlargement in the reduction of intracanal bacteria. J Endod 2007; 33(1):21-3.

16. Saiter L, Barroso JM, Roldi A, Pereira RS, Intra JBG, Ribeiro FC, Pereira GS. Diâmetro anatômico e alargamento cervical: uma visão crítica sobre suas influências no preparo da região apical dos canais radiculares. Rev Bras de Pesq em Saúde 2011;13(3):73-9

17. Aguiar CM, Câmara AC. Avaliação das alterações morfológicas do terço apical em canais instrumentados com o ProTaper manual, Protaper e RaCe. Odontol Clin Cientif 2006; 5(3):211-5.

18. Machado MEL, Machado MLBBL, Antoniazzi JH. Eficácia da técnica seriada convencional e das técnicas escalonadas ápico-cervical e cévico-apical no preparo químico cirúrgico de canais curvos. Rev Bras de Odontol 1998; 55(2):72-5.

19. López FU, Fachin EV, Fontanella VRC, Barletta FB, Só MVR, Grecca FS. Apical transportation: a comparative evaluation of three root canal instrumentation techniques with three different apical diameters. J Endod 2008; 34(12):1545-8.

20. Tolomelli IT. Campos CN. Avaliação do preparo do canal radicular produzido por três sistemas motorizados de instrumentação, segundo a regularidade da conicidade. J Bras Endod 2005; 5(20): 381-6.

21. Aznar, FDC, Nishiyama CK, Hussne RP, Leonardo RT. Avaliação radiográfica do desvio apical após o preparo radicular utilizando o sistema Protaper. Endodontia 2006; 24:143-7.

22. Otoboni Filho JA, Holland R, Souza V, Bernabé PFE, Nery MJ, Dezem Júnior ED, Gomes Filho JE. Avaliação da preparação do canal com sistema de rotação alternada e diferentes limas de aço inoxidável. Rev Odontol Univ Cid São Paulo $2006 ; 18(3) 251-6$

\section{Endereço para correspondência}

Carmen Milena Rodrigues Siqueira Carvalho

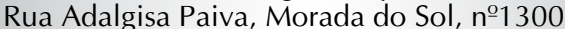
64056-105 Teresina-PI

Telefone: (86) 32329187

E-mail: carmenmilena@ufpi.br

Recebido: 27/05/2014. Aceito: 03/12/2014. 